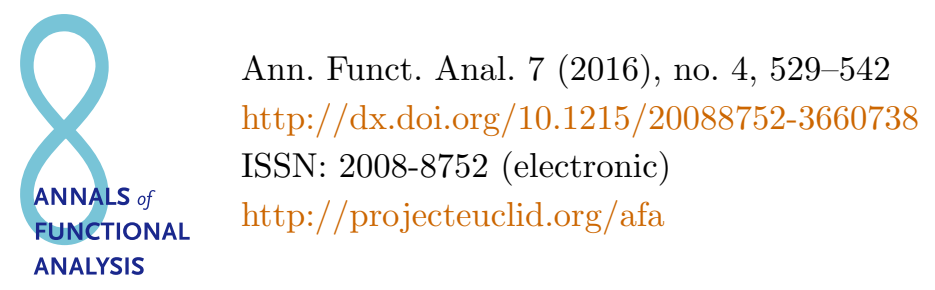

\title{
A PERTURBED EIGENVALUE PROBLEM ON GENERAL DOMAINS
}

\author{
MIHAI MIHĂILESCU ${ }^{1 *}$ and DENISA STANCU-DUMITRU ${ }^{2}$
}

Communicated by B. Ricceri

\begin{abstract}
The perturbed eigenvalue problem $-\Delta u-\Delta_{p} u=\lambda V(x) u$, with $p \in(1, N) \backslash\{2\}$ and $V$ a weight function which takes nonnegative values and may have singular points, is studied in an Orlicz-Sobolev setting on general open sets from $\mathbb{R}^{N}$ with $N \geq 3$. The analysis of these problems leads to a full characterization of the set of parameters $\lambda$ for which the problem possesses nontrivial solutions as being an unbounded open interval.
\end{abstract}

\section{INTRODUCTION AND MAIN RESULTS}

Let $\Omega \subseteq \mathbb{R}^{N}(N \geq 3)$ be an open set, and let $V: \Omega \rightarrow[0, \infty)$ be a function which satisfies the hypotheses

$$
\left\{\begin{array}{l}
V \in L_{\mathrm{loc}}^{1}(\Omega), V=V_{1}+V_{2}, V_{1} \in L^{N / 2}(\Omega), \\
\lim _{|x| \rightarrow \infty}|x|^{2} V_{2}(x)=0, \lim _{x \rightarrow y}|x-y|^{2} V_{2}(x)=0 \text { for any } y \in \bar{\Omega} .
\end{array}\right.
$$

For example, a weight function $V$ which satisfies condition (1.1) could be $V(x)=$ $|x|^{-2}\left(1+|x|^{2}\right)^{-1}\left[\log \left(2+1 /|x|^{2}\right)\right]^{-2 / N}$. (Other examples can be found in [6, Section 3].)

In [6], Szulkin and Willem analyzed the eigenvalue problem

$$
-\Delta u=\lambda V(x) u, \quad u \in \mathcal{D}_{0}^{1,2}(\Omega),
$$

Copyright 2016 by the Tusi Mathematical Research Group.

Received Jan. 10, 2016; Accepted Mar. 11, 2016.

${ }^{*}$ Corresponding author.

2010 Mathematics Subject Classification. Primary 35J60; Secondary 35J92, 46E30, 49R05.

Keywords. perturbed eigenvalue problem, Orlicz-Sobolev space, Nehari manifold, variational methods. 


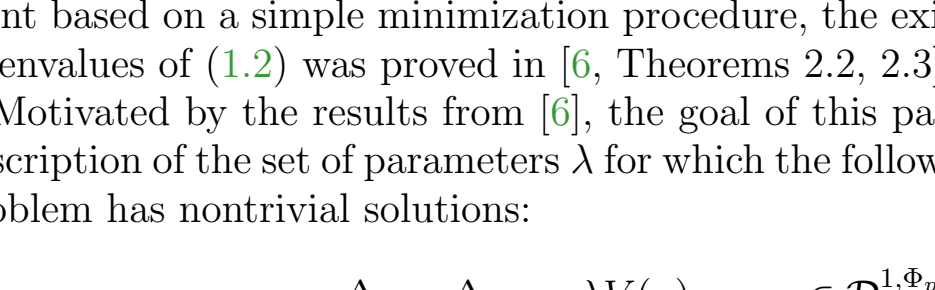

\title{
A PERTURBED EIGENVALUE PROBLEM ON GENERAL DOMAINS
}

\author{
MIHAI MIHĂILESCU ${ }^{1 *}$ and DENISA STANCU-DUMITRU ${ }^{2}$
}

Communicated by B. Ricceri

\begin{abstract}
The perturbed eigenvalue problem $-\Delta u-\Delta_{p} u=\lambda V(x) u$, with $p \in(1, N) \backslash\{2\}$ and $V$ a weight function which takes nonnegative values and may have singular points, is studied in an Orlicz-Sobolev setting on general open sets from $\mathbb{R}^{N}$ with $N \geq 3$. The analysis of these problems leads to a full characterization of the set of parameters $\lambda$ for which the problem possesses nontrivial solutions as being an unbounded open interval.
\end{abstract}

\section{INTRODUCTION AND MAIN RESULTS}

Let $\Omega \subseteq \mathbb{R}^{N}(N \geq 3)$ be an open set, and let $V: \Omega \rightarrow[0, \infty)$ be a function which satisfies the hypotheses

$$
\left\{\begin{array}{l}
V \in L_{\mathrm{loc}}^{1}(\Omega), V=V_{1}+V_{2}, V_{1} \in L^{N / 2}(\Omega), \\
\lim _{|x| \rightarrow \infty}|x|^{2} V_{2}(x)=0, \lim _{x \rightarrow y}|x-y|^{2} V_{2}(x)=0 \text { for any } y \in \bar{\Omega} .
\end{array}\right.
$$

For example, a weight function $V$ which satisfies condition (1.1) could be $V(x)=$ $|x|^{-2}\left(1+|x|^{2}\right)^{-1}\left[\log \left(2+1 /|x|^{2}\right)\right]^{-2 / N}$. (Other examples can be found in [6, Section 3].)

In [6], Szulkin and Willem analyzed the eigenvalue problem

$$
-\Delta u=\lambda V(x) u, \quad u \in \mathcal{D}_{0}^{1,2}(\Omega),
$$

Copyright 2016 by the Tusi Mathematical Research Group.

Received Jan. 10, 2016; Accepted Mar. 11, 2016.

${ }^{*}$ Corresponding author.

2010 Mathematics Subject Classification. Primary 35J60; Secondary 35J92, 46E30, 49R05.

Keywords. perturbed eigenvalue problem, Orlicz-Sobolev space, Nehari manifold, variational methods. 


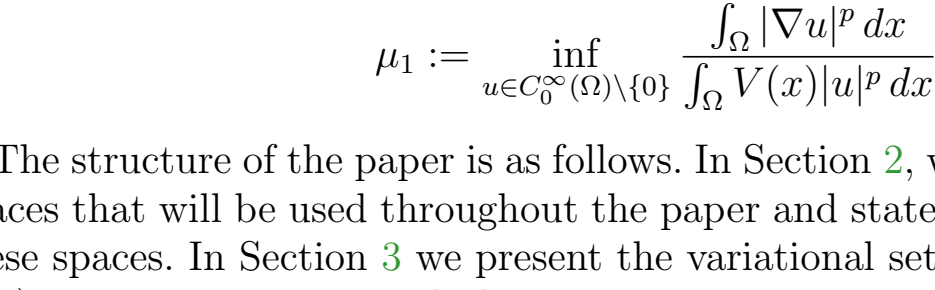

\title{
A PERTURBED EIGENVALUE PROBLEM ON GENERAL DOMAINS
}

\author{
MIHAI MIHĂILESCU ${ }^{1 *}$ and DENISA STANCU-DUMITRU ${ }^{2}$
}

Communicated by B. Ricceri

\begin{abstract}
The perturbed eigenvalue problem $-\Delta u-\Delta_{p} u=\lambda V(x) u$, with $p \in(1, N) \backslash\{2\}$ and $V$ a weight function which takes nonnegative values and may have singular points, is studied in an Orlicz-Sobolev setting on general open sets from $\mathbb{R}^{N}$ with $N \geq 3$. The analysis of these problems leads to a full characterization of the set of parameters $\lambda$ for which the problem possesses nontrivial solutions as being an unbounded open interval.
\end{abstract}

\section{INTRODUCTION AND MAIN RESULTS}

Let $\Omega \subseteq \mathbb{R}^{N}(N \geq 3)$ be an open set, and let $V: \Omega \rightarrow[0, \infty)$ be a function which satisfies the hypotheses

$$
\left\{\begin{array}{l}
V \in L_{\mathrm{loc}}^{1}(\Omega), V=V_{1}+V_{2}, V_{1} \in L^{N / 2}(\Omega), \\
\lim _{|x| \rightarrow \infty}|x|^{2} V_{2}(x)=0, \lim _{x \rightarrow y}|x-y|^{2} V_{2}(x)=0 \text { for any } y \in \bar{\Omega} .
\end{array}\right.
$$

For example, a weight function $V$ which satisfies condition (1.1) could be $V(x)=$ $|x|^{-2}\left(1+|x|^{2}\right)^{-1}\left[\log \left(2+1 /|x|^{2}\right)\right]^{-2 / N}$. (Other examples can be found in [6, Section 3].)

In [6], Szulkin and Willem analyzed the eigenvalue problem

$$
-\Delta u=\lambda V(x) u, \quad u \in \mathcal{D}_{0}^{1,2}(\Omega),
$$

Copyright 2016 by the Tusi Mathematical Research Group.

Received Jan. 10, 2016; Accepted Mar. 11, 2016.

${ }^{*}$ Corresponding author.

2010 Mathematics Subject Classification. Primary 35J60; Secondary 35J92, 46E30, 49R05.

Keywords. perturbed eigenvalue problem, Orlicz-Sobolev space, Nehari manifold, variational methods. 


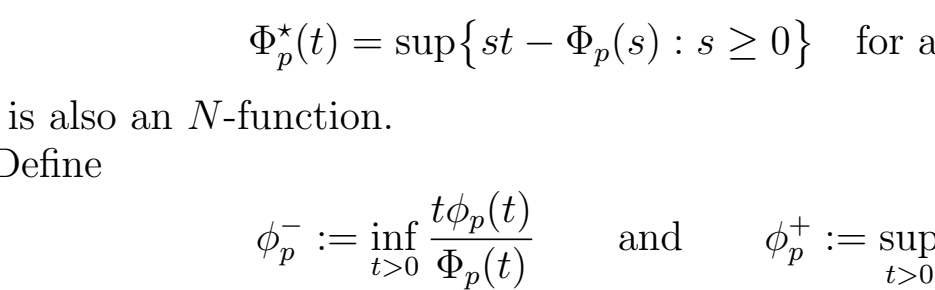

\title{
A PERTURBED EIGENVALUE PROBLEM ON GENERAL DOMAINS
}

\author{
MIHAI MIHĂILESCU ${ }^{1 *}$ and DENISA STANCU-DUMITRU ${ }^{2}$
}

Communicated by B. Ricceri

\begin{abstract}
The perturbed eigenvalue problem $-\Delta u-\Delta_{p} u=\lambda V(x) u$, with $p \in(1, N) \backslash\{2\}$ and $V$ a weight function which takes nonnegative values and may have singular points, is studied in an Orlicz-Sobolev setting on general open sets from $\mathbb{R}^{N}$ with $N \geq 3$. The analysis of these problems leads to a full characterization of the set of parameters $\lambda$ for which the problem possesses nontrivial solutions as being an unbounded open interval.
\end{abstract}

\section{INTRODUCTION AND MAIN RESULTS}

Let $\Omega \subseteq \mathbb{R}^{N}(N \geq 3)$ be an open set, and let $V: \Omega \rightarrow[0, \infty)$ be a function which satisfies the hypotheses

$$
\left\{\begin{array}{l}
V \in L_{\mathrm{loc}}^{1}(\Omega), V=V_{1}+V_{2}, V_{1} \in L^{N / 2}(\Omega), \\
\lim _{|x| \rightarrow \infty}|x|^{2} V_{2}(x)=0, \lim _{x \rightarrow y}|x-y|^{2} V_{2}(x)=0 \text { for any } y \in \bar{\Omega} .
\end{array}\right.
$$

For example, a weight function $V$ which satisfies condition (1.1) could be $V(x)=$ $|x|^{-2}\left(1+|x|^{2}\right)^{-1}\left[\log \left(2+1 /|x|^{2}\right)\right]^{-2 / N}$. (Other examples can be found in [6, Section 3].)

In [6], Szulkin and Willem analyzed the eigenvalue problem

$$
-\Delta u=\lambda V(x) u, \quad u \in \mathcal{D}_{0}^{1,2}(\Omega),
$$

Copyright 2016 by the Tusi Mathematical Research Group.

Received Jan. 10, 2016; Accepted Mar. 11, 2016.

${ }^{*}$ Corresponding author.

2010 Mathematics Subject Classification. Primary 35J60; Secondary 35J92, 46E30, 49R05.

Keywords. perturbed eigenvalue problem, Orlicz-Sobolev space, Nehari manifold, variational methods. 


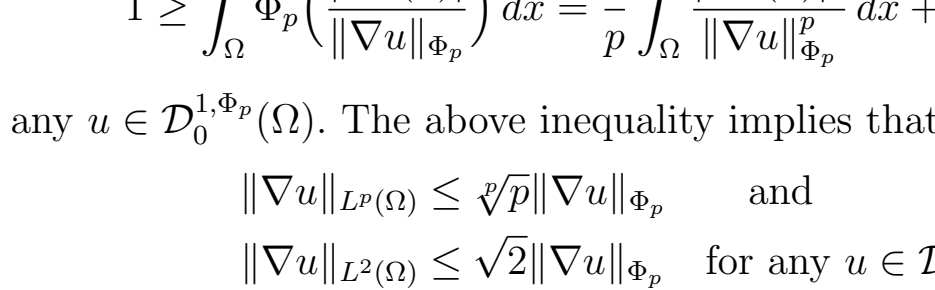

\title{
A PERTURBED EIGENVALUE PROBLEM ON GENERAL DOMAINS
}

\author{
MIHAI MIHĂILESCU ${ }^{1 *}$ and DENISA STANCU-DUMITRU ${ }^{2}$
}

Communicated by B. Ricceri

\begin{abstract}
The perturbed eigenvalue problem $-\Delta u-\Delta_{p} u=\lambda V(x) u$, with $p \in(1, N) \backslash\{2\}$ and $V$ a weight function which takes nonnegative values and may have singular points, is studied in an Orlicz-Sobolev setting on general open sets from $\mathbb{R}^{N}$ with $N \geq 3$. The analysis of these problems leads to a full characterization of the set of parameters $\lambda$ for which the problem possesses nontrivial solutions as being an unbounded open interval.
\end{abstract}

\section{INTRODUCTION AND MAIN RESULTS}

Let $\Omega \subseteq \mathbb{R}^{N}(N \geq 3)$ be an open set, and let $V: \Omega \rightarrow[0, \infty)$ be a function which satisfies the hypotheses

$$
\left\{\begin{array}{l}
V \in L_{\mathrm{loc}}^{1}(\Omega), V=V_{1}+V_{2}, V_{1} \in L^{N / 2}(\Omega), \\
\lim _{|x| \rightarrow \infty}|x|^{2} V_{2}(x)=0, \lim _{x \rightarrow y}|x-y|^{2} V_{2}(x)=0 \text { for any } y \in \bar{\Omega} .
\end{array}\right.
$$

For example, a weight function $V$ which satisfies condition (1.1) could be $V(x)=$ $|x|^{-2}\left(1+|x|^{2}\right)^{-1}\left[\log \left(2+1 /|x|^{2}\right)\right]^{-2 / N}$. (Other examples can be found in [6, Section 3].)

In [6], Szulkin and Willem analyzed the eigenvalue problem

$$
-\Delta u=\lambda V(x) u, \quad u \in \mathcal{D}_{0}^{1,2}(\Omega),
$$

Copyright 2016 by the Tusi Mathematical Research Group.

Received Jan. 10, 2016; Accepted Mar. 11, 2016.

${ }^{*}$ Corresponding author.

2010 Mathematics Subject Classification. Primary 35J60; Secondary 35J92, 46E30, 49R05.

Keywords. perturbed eigenvalue problem, Orlicz-Sobolev space, Nehari manifold, variational methods. 


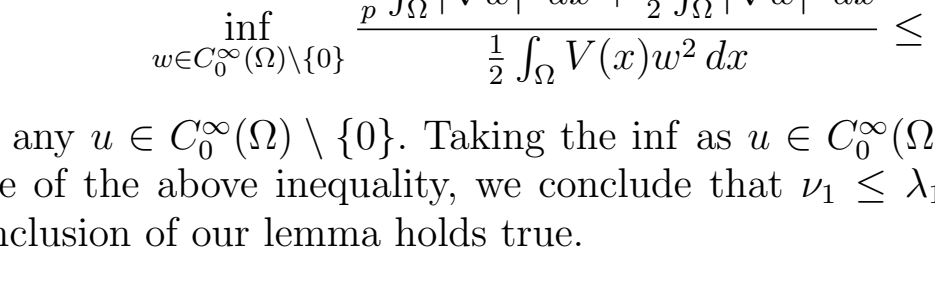

\title{
A PERTURBED EIGENVALUE PROBLEM ON GENERAL DOMAINS
}

\author{
MIHAI MIHĂILESCU ${ }^{1 *}$ and DENISA STANCU-DUMITRU ${ }^{2}$
}

Communicated by B. Ricceri

\begin{abstract}
The perturbed eigenvalue problem $-\Delta u-\Delta_{p} u=\lambda V(x) u$, with $p \in(1, N) \backslash\{2\}$ and $V$ a weight function which takes nonnegative values and may have singular points, is studied in an Orlicz-Sobolev setting on general open sets from $\mathbb{R}^{N}$ with $N \geq 3$. The analysis of these problems leads to a full characterization of the set of parameters $\lambda$ for which the problem possesses nontrivial solutions as being an unbounded open interval.
\end{abstract}

\section{INTRODUCTION AND MAIN RESULTS}

Let $\Omega \subseteq \mathbb{R}^{N}(N \geq 3)$ be an open set, and let $V: \Omega \rightarrow[0, \infty)$ be a function which satisfies the hypotheses

$$
\left\{\begin{array}{l}
V \in L_{\mathrm{loc}}^{1}(\Omega), V=V_{1}+V_{2}, V_{1} \in L^{N / 2}(\Omega), \\
\lim _{|x| \rightarrow \infty}|x|^{2} V_{2}(x)=0, \lim _{x \rightarrow y}|x-y|^{2} V_{2}(x)=0 \text { for any } y \in \bar{\Omega} .
\end{array}\right.
$$

For example, a weight function $V$ which satisfies condition (1.1) could be $V(x)=$ $|x|^{-2}\left(1+|x|^{2}\right)^{-1}\left[\log \left(2+1 /|x|^{2}\right)\right]^{-2 / N}$. (Other examples can be found in [6, Section 3].)

In [6], Szulkin and Willem analyzed the eigenvalue problem

$$
-\Delta u=\lambda V(x) u, \quad u \in \mathcal{D}_{0}^{1,2}(\Omega),
$$

Copyright 2016 by the Tusi Mathematical Research Group.

Received Jan. 10, 2016; Accepted Mar. 11, 2016.

${ }^{*}$ Corresponding author.

2010 Mathematics Subject Classification. Primary 35J60; Secondary 35J92, 46E30, 49R05.

Keywords. perturbed eigenvalue problem, Orlicz-Sobolev space, Nehari manifold, variational methods. 


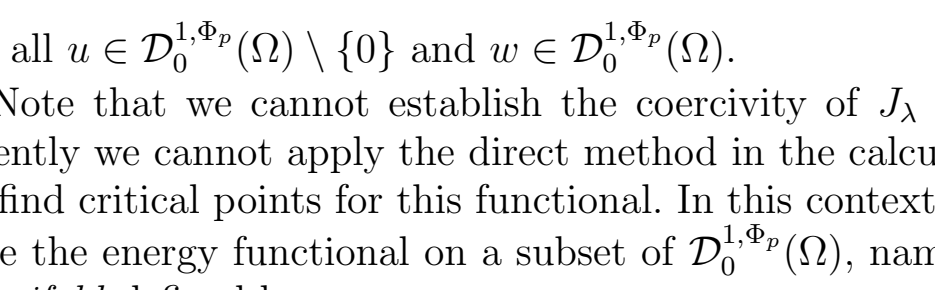

\title{
A PERTURBED EIGENVALUE PROBLEM ON GENERAL DOMAINS
}

\author{
MIHAI MIHĂILESCU ${ }^{1 *}$ and DENISA STANCU-DUMITRU ${ }^{2}$
}

Communicated by B. Ricceri

\begin{abstract}
The perturbed eigenvalue problem $-\Delta u-\Delta_{p} u=\lambda V(x) u$, with $p \in(1, N) \backslash\{2\}$ and $V$ a weight function which takes nonnegative values and may have singular points, is studied in an Orlicz-Sobolev setting on general open sets from $\mathbb{R}^{N}$ with $N \geq 3$. The analysis of these problems leads to a full characterization of the set of parameters $\lambda$ for which the problem possesses nontrivial solutions as being an unbounded open interval.
\end{abstract}

\section{INTRODUCTION AND MAIN RESULTS}

Let $\Omega \subseteq \mathbb{R}^{N}(N \geq 3)$ be an open set, and let $V: \Omega \rightarrow[0, \infty)$ be a function which satisfies the hypotheses

$$
\left\{\begin{array}{l}
V \in L_{\mathrm{loc}}^{1}(\Omega), V=V_{1}+V_{2}, V_{1} \in L^{N / 2}(\Omega), \\
\lim _{|x| \rightarrow \infty}|x|^{2} V_{2}(x)=0, \lim _{x \rightarrow y}|x-y|^{2} V_{2}(x)=0 \text { for any } y \in \bar{\Omega} .
\end{array}\right.
$$

For example, a weight function $V$ which satisfies condition (1.1) could be $V(x)=$ $|x|^{-2}\left(1+|x|^{2}\right)^{-1}\left[\log \left(2+1 /|x|^{2}\right)\right]^{-2 / N}$. (Other examples can be found in [6, Section 3].)

In [6], Szulkin and Willem analyzed the eigenvalue problem

$$
-\Delta u=\lambda V(x) u, \quad u \in \mathcal{D}_{0}^{1,2}(\Omega),
$$

Copyright 2016 by the Tusi Mathematical Research Group.

Received Jan. 10, 2016; Accepted Mar. 11, 2016.

${ }^{*}$ Corresponding author.

2010 Mathematics Subject Classification. Primary 35J60; Secondary 35J92, 46E30, 49R05.

Keywords. perturbed eigenvalue problem, Orlicz-Sobolev space, Nehari manifold, variational methods. 


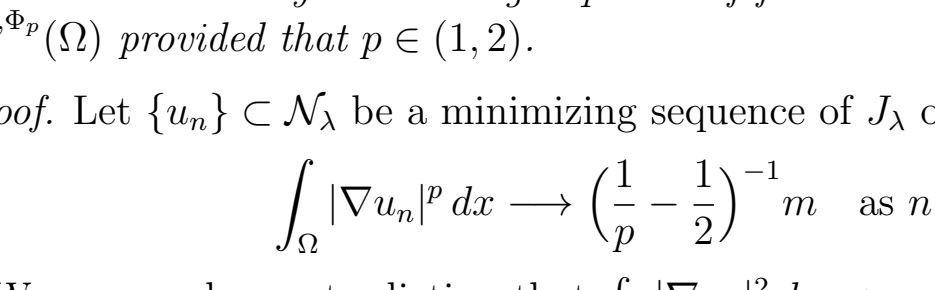

\title{
A PERTURBED EIGENVALUE PROBLEM ON GENERAL DOMAINS
}

\author{
MIHAI MIHĂILESCU ${ }^{1 *}$ and DENISA STANCU-DUMITRU ${ }^{2}$
}

Communicated by B. Ricceri

\begin{abstract}
The perturbed eigenvalue problem $-\Delta u-\Delta_{p} u=\lambda V(x) u$, with $p \in(1, N) \backslash\{2\}$ and $V$ a weight function which takes nonnegative values and may have singular points, is studied in an Orlicz-Sobolev setting on general open sets from $\mathbb{R}^{N}$ with $N \geq 3$. The analysis of these problems leads to a full characterization of the set of parameters $\lambda$ for which the problem possesses nontrivial solutions as being an unbounded open interval.
\end{abstract}

\section{INTRODUCTION AND MAIN RESULTS}

Let $\Omega \subseteq \mathbb{R}^{N}(N \geq 3)$ be an open set, and let $V: \Omega \rightarrow[0, \infty)$ be a function which satisfies the hypotheses

$$
\left\{\begin{array}{l}
V \in L_{\mathrm{loc}}^{1}(\Omega), V=V_{1}+V_{2}, V_{1} \in L^{N / 2}(\Omega), \\
\lim _{|x| \rightarrow \infty}|x|^{2} V_{2}(x)=0, \lim _{x \rightarrow y}|x-y|^{2} V_{2}(x)=0 \text { for any } y \in \bar{\Omega} .
\end{array}\right.
$$

For example, a weight function $V$ which satisfies condition (1.1) could be $V(x)=$ $|x|^{-2}\left(1+|x|^{2}\right)^{-1}\left[\log \left(2+1 /|x|^{2}\right)\right]^{-2 / N}$. (Other examples can be found in [6, Section 3].)

In [6], Szulkin and Willem analyzed the eigenvalue problem

$$
-\Delta u=\lambda V(x) u, \quad u \in \mathcal{D}_{0}^{1,2}(\Omega),
$$

Copyright 2016 by the Tusi Mathematical Research Group.

Received Jan. 10, 2016; Accepted Mar. 11, 2016.

${ }^{*}$ Corresponding author.

2010 Mathematics Subject Classification. Primary 35J60; Secondary 35J92, 46E30, 49R05.

Keywords. perturbed eigenvalue problem, Orlicz-Sobolev space, Nehari manifold, variational methods. 


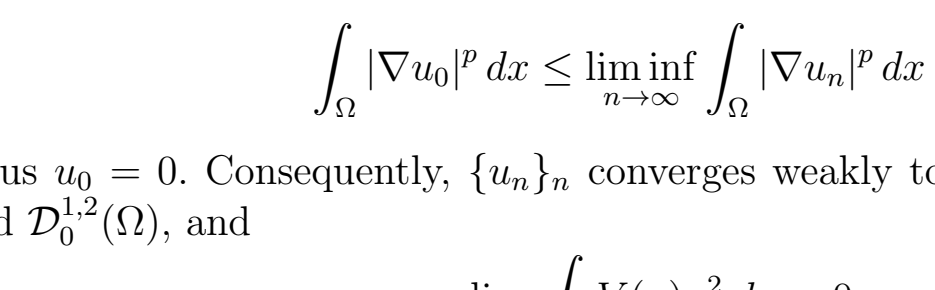

\title{
A PERTURBED EIGENVALUE PROBLEM ON GENERAL DOMAINS
}

\author{
MIHAI MIHĂILESCU ${ }^{1 *}$ and DENISA STANCU-DUMITRU ${ }^{2}$
}

Communicated by B. Ricceri

\begin{abstract}
The perturbed eigenvalue problem $-\Delta u-\Delta_{p} u=\lambda V(x) u$, with $p \in(1, N) \backslash\{2\}$ and $V$ a weight function which takes nonnegative values and may have singular points, is studied in an Orlicz-Sobolev setting on general open sets from $\mathbb{R}^{N}$ with $N \geq 3$. The analysis of these problems leads to a full characterization of the set of parameters $\lambda$ for which the problem possesses nontrivial solutions as being an unbounded open interval.
\end{abstract}

\section{INTRODUCTION AND MAIN RESULTS}

Let $\Omega \subseteq \mathbb{R}^{N}(N \geq 3)$ be an open set, and let $V: \Omega \rightarrow[0, \infty)$ be a function which satisfies the hypotheses

$$
\left\{\begin{array}{l}
V \in L_{\mathrm{loc}}^{1}(\Omega), V=V_{1}+V_{2}, V_{1} \in L^{N / 2}(\Omega), \\
\lim _{|x| \rightarrow \infty}|x|^{2} V_{2}(x)=0, \lim _{x \rightarrow y}|x-y|^{2} V_{2}(x)=0 \text { for any } y \in \bar{\Omega} .
\end{array}\right.
$$

For example, a weight function $V$ which satisfies condition (1.1) could be $V(x)=$ $|x|^{-2}\left(1+|x|^{2}\right)^{-1}\left[\log \left(2+1 /|x|^{2}\right)\right]^{-2 / N}$. (Other examples can be found in [6, Section 3].)

In [6], Szulkin and Willem analyzed the eigenvalue problem

$$
-\Delta u=\lambda V(x) u, \quad u \in \mathcal{D}_{0}^{1,2}(\Omega),
$$

Copyright 2016 by the Tusi Mathematical Research Group.

Received Jan. 10, 2016; Accepted Mar. 11, 2016.

${ }^{*}$ Corresponding author.

2010 Mathematics Subject Classification. Primary 35J60; Secondary 35J92, 46E30, 49R05.

Keywords. perturbed eigenvalue problem, Orlicz-Sobolev space, Nehari manifold, variational methods. 


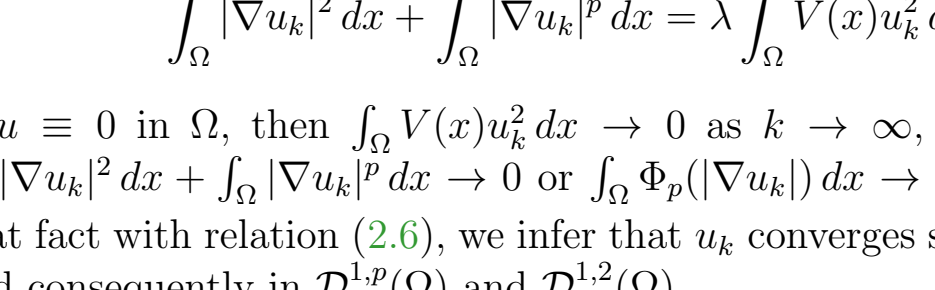

\title{
A PERTURBED EIGENVALUE PROBLEM ON GENERAL DOMAINS
}

\author{
MIHAI MIHĂILESCU ${ }^{1 *}$ and DENISA STANCU-DUMITRU ${ }^{2}$
}

Communicated by B. Ricceri

\begin{abstract}
The perturbed eigenvalue problem $-\Delta u-\Delta_{p} u=\lambda V(x) u$, with $p \in(1, N) \backslash\{2\}$ and $V$ a weight function which takes nonnegative values and may have singular points, is studied in an Orlicz-Sobolev setting on general open sets from $\mathbb{R}^{N}$ with $N \geq 3$. The analysis of these problems leads to a full characterization of the set of parameters $\lambda$ for which the problem possesses nontrivial solutions as being an unbounded open interval.
\end{abstract}

\section{INTRODUCTION AND MAIN RESULTS}

Let $\Omega \subseteq \mathbb{R}^{N}(N \geq 3)$ be an open set, and let $V: \Omega \rightarrow[0, \infty)$ be a function which satisfies the hypotheses

$$
\left\{\begin{array}{l}
V \in L_{\mathrm{loc}}^{1}(\Omega), V=V_{1}+V_{2}, V_{1} \in L^{N / 2}(\Omega), \\
\lim _{|x| \rightarrow \infty}|x|^{2} V_{2}(x)=0, \lim _{x \rightarrow y}|x-y|^{2} V_{2}(x)=0 \text { for any } y \in \bar{\Omega} .
\end{array}\right.
$$

For example, a weight function $V$ which satisfies condition (1.1) could be $V(x)=$ $|x|^{-2}\left(1+|x|^{2}\right)^{-1}\left[\log \left(2+1 /|x|^{2}\right)\right]^{-2 / N}$. (Other examples can be found in [6, Section 3].)

In [6], Szulkin and Willem analyzed the eigenvalue problem

$$
-\Delta u=\lambda V(x) u, \quad u \in \mathcal{D}_{0}^{1,2}(\Omega),
$$

Copyright 2016 by the Tusi Mathematical Research Group.

Received Jan. 10, 2016; Accepted Mar. 11, 2016.

${ }^{*}$ Corresponding author.

2010 Mathematics Subject Classification. Primary 35J60; Secondary 35J92, 46E30, 49R05.

Keywords. perturbed eigenvalue problem, Orlicz-Sobolev space, Nehari manifold, variational methods. 


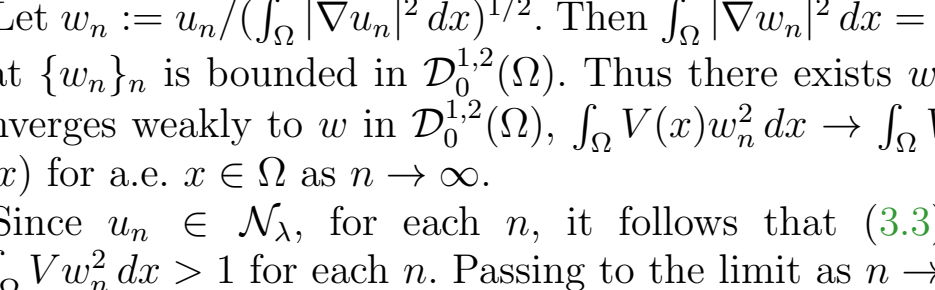

\title{
A PERTURBED EIGENVALUE PROBLEM ON GENERAL DOMAINS
}

\author{
MIHAI MIHĂILESCU ${ }^{1 *}$ and DENISA STANCU-DUMITRU ${ }^{2}$
}

Communicated by B. Ricceri

\begin{abstract}
The perturbed eigenvalue problem $-\Delta u-\Delta_{p} u=\lambda V(x) u$, with $p \in(1, N) \backslash\{2\}$ and $V$ a weight function which takes nonnegative values and may have singular points, is studied in an Orlicz-Sobolev setting on general open sets from $\mathbb{R}^{N}$ with $N \geq 3$. The analysis of these problems leads to a full characterization of the set of parameters $\lambda$ for which the problem possesses nontrivial solutions as being an unbounded open interval.
\end{abstract}

\section{INTRODUCTION AND MAIN RESULTS}

Let $\Omega \subseteq \mathbb{R}^{N}(N \geq 3)$ be an open set, and let $V: \Omega \rightarrow[0, \infty)$ be a function which satisfies the hypotheses

$$
\left\{\begin{array}{l}
V \in L_{\mathrm{loc}}^{1}(\Omega), V=V_{1}+V_{2}, V_{1} \in L^{N / 2}(\Omega), \\
\lim _{|x| \rightarrow \infty}|x|^{2} V_{2}(x)=0, \lim _{x \rightarrow y}|x-y|^{2} V_{2}(x)=0 \text { for any } y \in \bar{\Omega} .
\end{array}\right.
$$

For example, a weight function $V$ which satisfies condition (1.1) could be $V(x)=$ $|x|^{-2}\left(1+|x|^{2}\right)^{-1}\left[\log \left(2+1 /|x|^{2}\right)\right]^{-2 / N}$. (Other examples can be found in [6, Section 3].)

In [6], Szulkin and Willem analyzed the eigenvalue problem

$$
-\Delta u=\lambda V(x) u, \quad u \in \mathcal{D}_{0}^{1,2}(\Omega),
$$

Copyright 2016 by the Tusi Mathematical Research Group.

Received Jan. 10, 2016; Accepted Mar. 11, 2016.

${ }^{*}$ Corresponding author.

2010 Mathematics Subject Classification. Primary 35J60; Secondary 35J92, 46E30, 49R05.

Keywords. perturbed eigenvalue problem, Orlicz-Sobolev space, Nehari manifold, variational methods. 


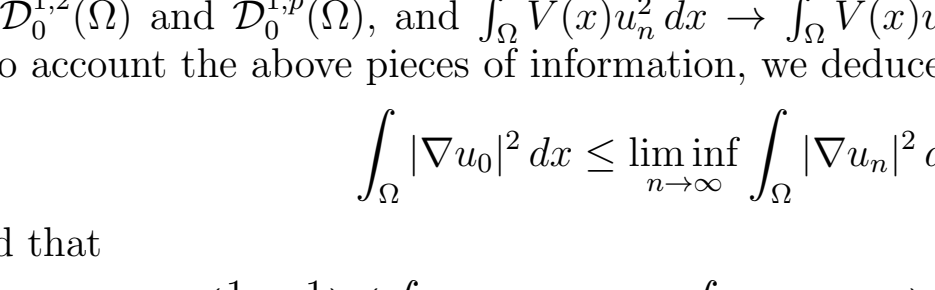

\title{
A PERTURBED EIGENVALUE PROBLEM ON GENERAL DOMAINS
}

\author{
MIHAI MIHĂILESCU ${ }^{1 *}$ and DENISA STANCU-DUMITRU ${ }^{2}$
}

Communicated by B. Ricceri

\begin{abstract}
The perturbed eigenvalue problem $-\Delta u-\Delta_{p} u=\lambda V(x) u$, with $p \in(1, N) \backslash\{2\}$ and $V$ a weight function which takes nonnegative values and may have singular points, is studied in an Orlicz-Sobolev setting on general open sets from $\mathbb{R}^{N}$ with $N \geq 3$. The analysis of these problems leads to a full characterization of the set of parameters $\lambda$ for which the problem possesses nontrivial solutions as being an unbounded open interval.
\end{abstract}

\section{INTRODUCTION AND MAIN RESULTS}

Let $\Omega \subseteq \mathbb{R}^{N}(N \geq 3)$ be an open set, and let $V: \Omega \rightarrow[0, \infty)$ be a function which satisfies the hypotheses

$$
\left\{\begin{array}{l}
V \in L_{\mathrm{loc}}^{1}(\Omega), V=V_{1}+V_{2}, V_{1} \in L^{N / 2}(\Omega), \\
\lim _{|x| \rightarrow \infty}|x|^{2} V_{2}(x)=0, \lim _{x \rightarrow y}|x-y|^{2} V_{2}(x)=0 \text { for any } y \in \bar{\Omega} .
\end{array}\right.
$$

For example, a weight function $V$ which satisfies condition (1.1) could be $V(x)=$ $|x|^{-2}\left(1+|x|^{2}\right)^{-1}\left[\log \left(2+1 /|x|^{2}\right)\right]^{-2 / N}$. (Other examples can be found in [6, Section 3].)

In [6], Szulkin and Willem analyzed the eigenvalue problem

$$
-\Delta u=\lambda V(x) u, \quad u \in \mathcal{D}_{0}^{1,2}(\Omega),
$$

Copyright 2016 by the Tusi Mathematical Research Group.

Received Jan. 10, 2016; Accepted Mar. 11, 2016.

${ }^{*}$ Corresponding author.

2010 Mathematics Subject Classification. Primary 35J60; Secondary 35J92, 46E30, 49R05.

Keywords. perturbed eigenvalue problem, Orlicz-Sobolev space, Nehari manifold, variational methods. 


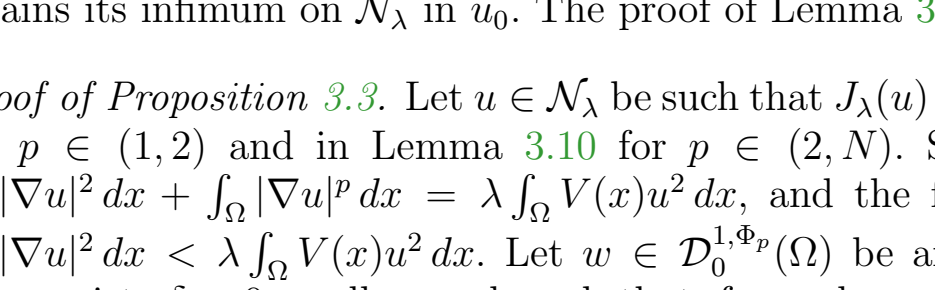

\title{
A PERTURBED EIGENVALUE PROBLEM ON GENERAL DOMAINS
}

\author{
MIHAI MIHĂILESCU ${ }^{1 *}$ and DENISA STANCU-DUMITRU ${ }^{2}$
}

Communicated by B. Ricceri

\begin{abstract}
The perturbed eigenvalue problem $-\Delta u-\Delta_{p} u=\lambda V(x) u$, with $p \in(1, N) \backslash\{2\}$ and $V$ a weight function which takes nonnegative values and may have singular points, is studied in an Orlicz-Sobolev setting on general open sets from $\mathbb{R}^{N}$ with $N \geq 3$. The analysis of these problems leads to a full characterization of the set of parameters $\lambda$ for which the problem possesses nontrivial solutions as being an unbounded open interval.
\end{abstract}

\section{INTRODUCTION AND MAIN RESULTS}

Let $\Omega \subseteq \mathbb{R}^{N}(N \geq 3)$ be an open set, and let $V: \Omega \rightarrow[0, \infty)$ be a function which satisfies the hypotheses

$$
\left\{\begin{array}{l}
V \in L_{\mathrm{loc}}^{1}(\Omega), V=V_{1}+V_{2}, V_{1} \in L^{N / 2}(\Omega), \\
\lim _{|x| \rightarrow \infty}|x|^{2} V_{2}(x)=0, \lim _{x \rightarrow y}|x-y|^{2} V_{2}(x)=0 \text { for any } y \in \bar{\Omega} .
\end{array}\right.
$$

For example, a weight function $V$ which satisfies condition (1.1) could be $V(x)=$ $|x|^{-2}\left(1+|x|^{2}\right)^{-1}\left[\log \left(2+1 /|x|^{2}\right)\right]^{-2 / N}$. (Other examples can be found in [6, Section 3].)

In [6], Szulkin and Willem analyzed the eigenvalue problem

$$
-\Delta u=\lambda V(x) u, \quad u \in \mathcal{D}_{0}^{1,2}(\Omega),
$$

Copyright 2016 by the Tusi Mathematical Research Group.

Received Jan. 10, 2016; Accepted Mar. 11, 2016.

${ }^{*}$ Corresponding author.

2010 Mathematics Subject Classification. Primary 35J60; Secondary 35J92, 46E30, 49R05.

Keywords. perturbed eigenvalue problem, Orlicz-Sobolev space, Nehari manifold, variational methods. 


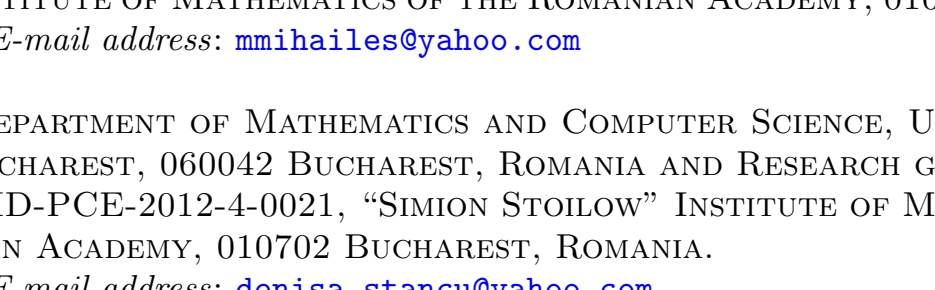

\title{
A PERTURBED EIGENVALUE PROBLEM ON GENERAL DOMAINS
}

\author{
MIHAI MIHĂILESCU ${ }^{1 *}$ and DENISA STANCU-DUMITRU ${ }^{2}$
}

Communicated by B. Ricceri

\begin{abstract}
The perturbed eigenvalue problem $-\Delta u-\Delta_{p} u=\lambda V(x) u$, with $p \in(1, N) \backslash\{2\}$ and $V$ a weight function which takes nonnegative values and may have singular points, is studied in an Orlicz-Sobolev setting on general open sets from $\mathbb{R}^{N}$ with $N \geq 3$. The analysis of these problems leads to a full characterization of the set of parameters $\lambda$ for which the problem possesses nontrivial solutions as being an unbounded open interval.
\end{abstract}

\section{INTRODUCTION AND MAIN RESULTS}

Let $\Omega \subseteq \mathbb{R}^{N}(N \geq 3)$ be an open set, and let $V: \Omega \rightarrow[0, \infty)$ be a function which satisfies the hypotheses

$$
\left\{\begin{array}{l}
V \in L_{\mathrm{loc}}^{1}(\Omega), V=V_{1}+V_{2}, V_{1} \in L^{N / 2}(\Omega), \\
\lim _{|x| \rightarrow \infty}|x|^{2} V_{2}(x)=0, \lim _{x \rightarrow y}|x-y|^{2} V_{2}(x)=0 \text { for any } y \in \bar{\Omega} .
\end{array}\right.
$$

For example, a weight function $V$ which satisfies condition (1.1) could be $V(x)=$ $|x|^{-2}\left(1+|x|^{2}\right)^{-1}\left[\log \left(2+1 /|x|^{2}\right)\right]^{-2 / N}$. (Other examples can be found in [6, Section 3].)

In [6], Szulkin and Willem analyzed the eigenvalue problem

$$
-\Delta u=\lambda V(x) u, \quad u \in \mathcal{D}_{0}^{1,2}(\Omega),
$$

Copyright 2016 by the Tusi Mathematical Research Group.

Received Jan. 10, 2016; Accepted Mar. 11, 2016.

${ }^{*}$ Corresponding author.

2010 Mathematics Subject Classification. Primary 35J60; Secondary 35J92, 46E30, 49R05.

Keywords. perturbed eigenvalue problem, Orlicz-Sobolev space, Nehari manifold, variational methods. 